\title{
Whole-lung lavage for severe pulmonary alveolar proteinosis assisted by veno-venous extracorporeal membrane oxygenation: a case report
}

\author{
João P. Moreira, MD¹, Sofia Ferraz, MD¹, Cláudia Freitas, $\mathrm{MD}^{2}$, António Morais, $\mathrm{PhD}^{2}$, Roberto R. Albuquerque, PhD³, Carlos Fiuza, MD ${ }^{1}$
}

\begin{abstract}
JP Moreira, S Ferraz, C Freitas, A Morais, RR Albuquerque, C Fiuza. Whole-lung lavage for severe pulmonary alveolar proteinosis assisted by veno-venous extracorporeal membrane oxygenation: a case report. Can J Respir Ther 2019;55:9-12. doi: 10.29390/cjrt-2018-019.

Pulmonary alveolar proteinosis (PAP) is a rare pathology characterized by accumulation of phospholipoproteinaceous material within the alveoli. The evolution of PAP is variable and treatment modalities are limited. Pharmacological therapeutic targets are being actively developed, but whole-lung lavage (WLL), first described in the 1960s, remains the cornerstone of therapy. The preferential treatment for PAP in our center is sequential WLL, where each lung is separately and sequentially perfused with warmed saline. However, some patients do not tolerate single lung ventilation (SLV), as there is a greater risk of severe hypoxemia with this method. Extracorporeal membrane oxygenation (ECMO), referring to an extracorporeal circuit that directly oxygenates and removes carbon dioxide from the blood, may be considered in highly selected patients with severe respiratory failure who otherwise would not be able to undergo WLL. In this context, veno-venous ECMO is most often utilized. We describe a case of a 44-year-old male diagnosed with silicosis five years earlier who presented with severe hypoxemic respiratory failure not amenable to WLL under general anesthesia with SLV, which was successfully managed with ECMO-assisted WLL.
\end{abstract}

Key Words: extracorporeal membrane oxygenation; pulmonary alveolar proteinosis; whole-lung lavage

\section{INTRODUCTION}

First reported in 1958, pulmonary alveolar proteinosis (PAP) is an extremely rare disease characterized by accumulation of phospholipoproteinaceous material within the alveoli as a result of decreased protein clearance by pulmonary macrophages, leading to gas exchange impairment, dyspnea, and alveolar infiltrates [1]. The homeostasis of surfactant and granulocyte macrophage colony stimulating factor (GM-CSF) is correlated and plays a crucial role in the pathogenesis of the disorder.

The estimated prevalence of PAP is 6.2-6.9 per million in the general population and is similar in males and females. The mean age of diagnosis is 39 years for men and 35 for women. However, considering difficulties and delays in diagnosing this is likely a minimum estimate of true prevalence. These patients had significantly more comorbidities, health care utilization, and associated costs [2-4]. Autoimmune PAP due to antibodies against GM-CSF is responsible for about $90 \%$ of the cases; however, there are secondary (hematological or environmental) and congenital forms [5]. The clinical course ranges from spontaneous resolution to death from infections or progressive respiratory failure.

Pharmacological therapeutic targets are being actively developed, but whole-lung lavage (WLL), first described in the 1960s, remains the cornerstone of therapy [5-7]. The method used for WLL usually involves single lung ventilation (SLV) and sequential lavage of one lung at a time. This is associated with increased risk of hypoxemia [8].

Extracorporeal membrane oxygenation (ECMO), referring to an extracorporeal circuit that directly oxygenates and removes carbon dioxide from the blood, may be considered in highly selected patients with severe respiratory failure who otherwise would not be able to undergo WLL with conventional techniques $[9,10]$. In this context, venovenous
(VV)-ECMO is most often utilized in which blood is withdrawn from and returned to a central vein. Recently, there has been increasing interest in ECMO because of advances in extracorporeal technology with more efficient oxygenators and lower rates of complications [11].

We report a case of secondary PAP, associated with a severe left lung perfusion defect, presenting with severe hypoxemic respiratory failure not amenable to WLL under general anesthesia with SLV, which was successfully managed with ECMO-assisted WLL.

\section{ETHICS}

Ethics committee (Comissão de Ética para a Saúde do Centro Hospitalar de São João e Faculdade de Medicina da Universidade do Porto) approval to conduct this study was granted by the authors' institution. Informed consent was obtained from all participants.

\section{CASE REPORT}

We describe a case of a 44-year-old Caucasian male diagnosed with silicosis five years earlier. He was a heavy smoker and worked as a miner for seven years. His past medical history included successfully treated pulmonary tuberculosis three years ago. He presented with a 4-month history of progressive exertional dyspnea and hypoxemic respiratory failure ( $\mathrm{pO}, 51 \mathrm{mmHg}, \mathrm{FiO}, 21 \%$ ). Further investigation included pulmonary function tests, which showed a functional mixed pattern (forced vital capacity $52.3 \%$, forced expiratory volume in one second $46 \%$, Tiffeneau Index $61.41 \%$ ) (Figure 1), and a decreased carbon monoxide diffusing capacity of $49.2 \%$ was noticed (Figure 1). High-resolution computed tomography (HRCT) showed a "crazy-paving" appearance superimposed in a pseudotumoral silicosis pattern. A bronchoalveolar lavage

${ }^{1}$ Department of Anesthesiology, Centro Hospitalar São João, Porto, Portugal

${ }^{2}$ Department of Pneumology, Centro Hospitalar São João, Porto, Portugal

${ }^{3}$ Department of Intensive Care Medicine, Centro Hospitalar São João, Porto, Portugal

Correspondence: João P. Moreira, Centro Hospitalar São João, Department of Anesthesiology, Alameda Prof. Hernâni Monteiro, 4200-319 Porto, Portugal. Tel.: +351225512100; E-mail: joaopbmoreira@gmail.com

Published online at https://www.cjrt.ca on 05 December 2018

This open-access article is distributed under the terms of the Creative Commons Attribution Non-Commercial License (CC BY-NC) (http:// creativecommons.org/licenses/by-nc/4.0/), which permits reuse, distribution and reproduction of the article, provided that the original work is properly cited and the reuse is restricted to noncommercial purposes. For commercial reuse, contact editor@csrt.com 
was performed and a Periodic acid-Schiff positive lipoproteinaceous material was detected. The presence of serum anti-GM-CSF antibodies suggested an autoimmune background; however, the presence of a significant silica exposure should be considered at least as potential trigger for the disease. Since the HRCT scan showed bilateral consolidations (Figure 2), with significant predominance in left lung, a scintigraphy was performed that revealed severe functional asymmetry with a left-toright perfusion relationship of 19:81\% (Figure 3). Because of the progressive worsening of exertional dyspnea, severe hypoxemia, and asymmetric ventilation-perfusion not allowing SLV, ECMO-assisted WLL was proposed to the patient.

\section{FIGURE 1}

Lung Function mixed pattern: forced vital Capacity $52.3 \%$, forced expiratory volume in one second $46 \%$, and Tiffeneau Index $61.4 \%$.

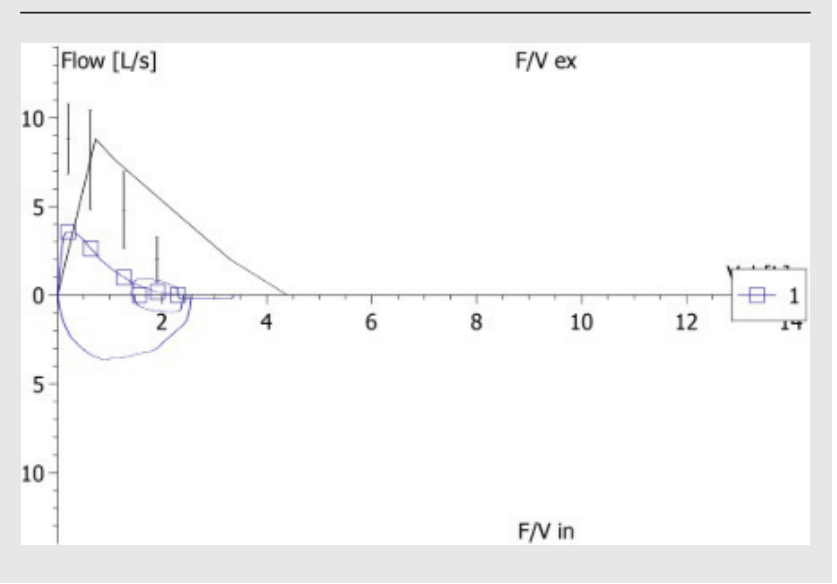

After noninvasive monitoring with electrocardiograph, noninvasive blood pressure measurement, and pulse oximetry, the patient underwent total intravenous anesthesia with propofol and fentanyl; muscular relaxation was achieved using rocuronium. Selective intubation was performed with a 39F left double-lumen endobronchial tube introduced into the left mainstem bronchus controlled by bronchofibroscopy. Radial arterial and jugular venous catheters were placed and enabled continuous hemodynamic monitoring. A bolus of heparin (2000 I.U.) was administered prior to cannulation for the VV-ECMO. The infusion $(50-\mathrm{cm}-\mathrm{long}$ 21-Fr; Medtronic, Minneapolis, USA) and drainage (55-cm-long 25-Fr; Maquet-Cardiopulmonary-AG, Hirrlingen, Germany) cannulae were placed percutaneously by Seldinger technique in the right and left femoral veins, respectively. VV-ECMO was initiated using a miniaturized, compact ECMO circuit with continuous pressures and venous saturation monitoring (HLS Set Advanced 7.0; Maquet-Cardiopulmonary-AG), with an initial blood flow of $3.44 \mathrm{~L}$ per minute and a sweep gas (100\% oxygen) flow of $2.0 \mathrm{~L}$ per minute (Figure 3 ).

Pulmonary lavage was performed with instillation and drainage of $1000 \mathrm{~mL}$ of warmed saline $\left(37^{\circ} \mathrm{C}\right)$ in each lung at a time, through gravity with the patient being positioned in the reverse trendelenburg and trendelenburg positions, respectively. This procedure was repeated 6 times in the left lung and 14 times in the right lung. The fluid collected was initially white and cloudy, and it progressively became clearer. The $\mathrm{FiO}_{2}$ values on ECMO were determined according to the patient $\mathrm{PaO}_{2}$ and varied between 0.6 and 0.8 for the duration of the procedure. The left lung was rinsed with $2400 \mathrm{~mL}$ of warm saline with $510 \mathrm{~mL}$ being absorbed over 40 minutes, and the right lung was rinsed with $15,700 \mathrm{~mL}$ of warm saline with $600 \mathrm{~mL}$ being absorbed over 90 minutes.

During the 180 minutes of the procedure, several incidents of hypotension were registered, mostly related to vigorous pulmonary saline inflow. They were spontaneously reversed and did not require any intervention. Temperature and electrolyte levels were uneventfully monitored throughout the procedure. Diuresis was stimulated using furosemide $(10 \mathrm{mg})$.

\section{FIGURE 2}

High-resolution computed tomography scan showing bilateral consolidations, with a predominance on the left lung.

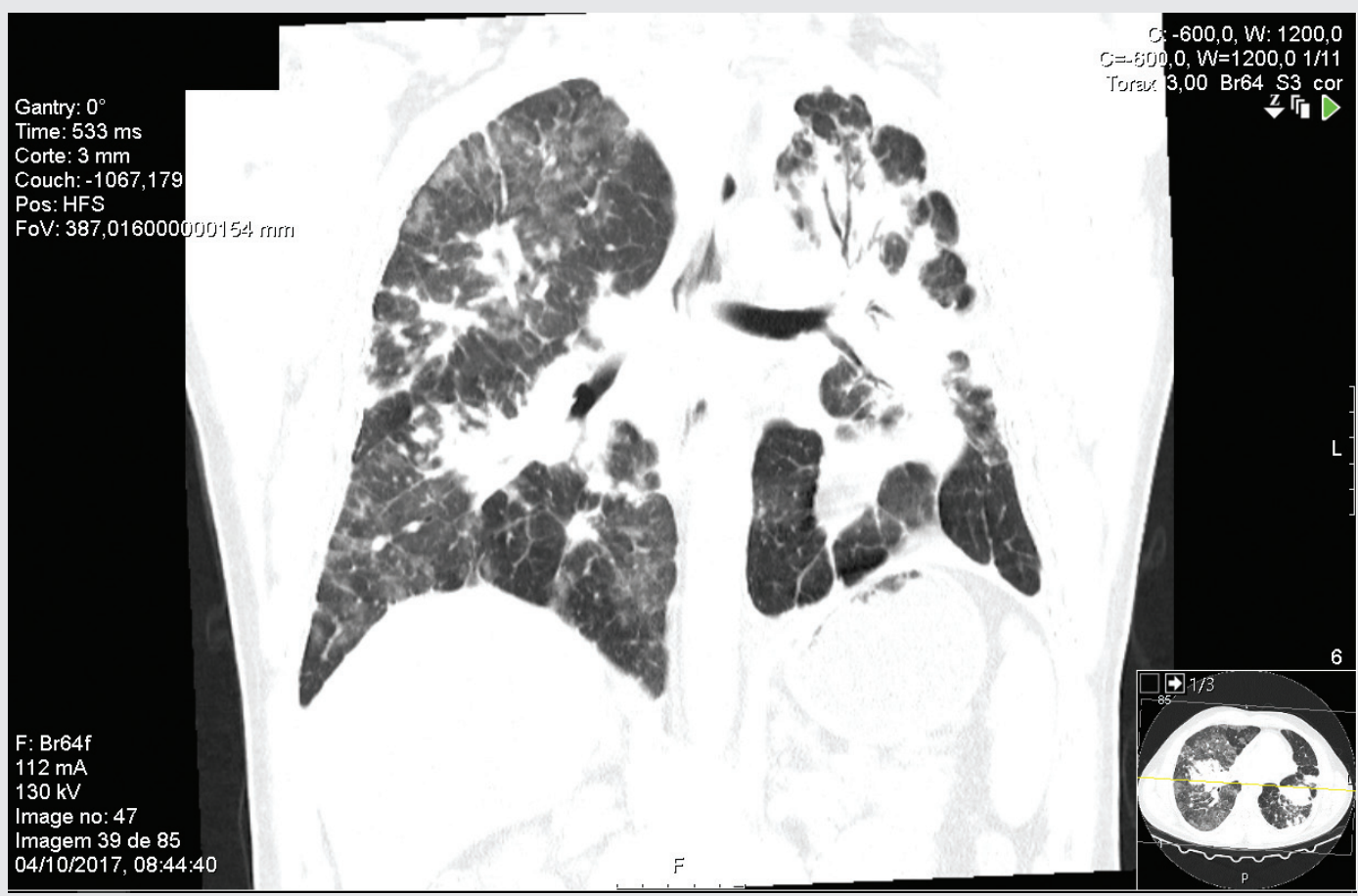




\section{FIGURE 3}

Perfusion lung scintigraphy showing severe functional asymmetry with left-to-right perfusion relationship of $19: 81 \%$, meaning an extremely poorly perfused left lung.

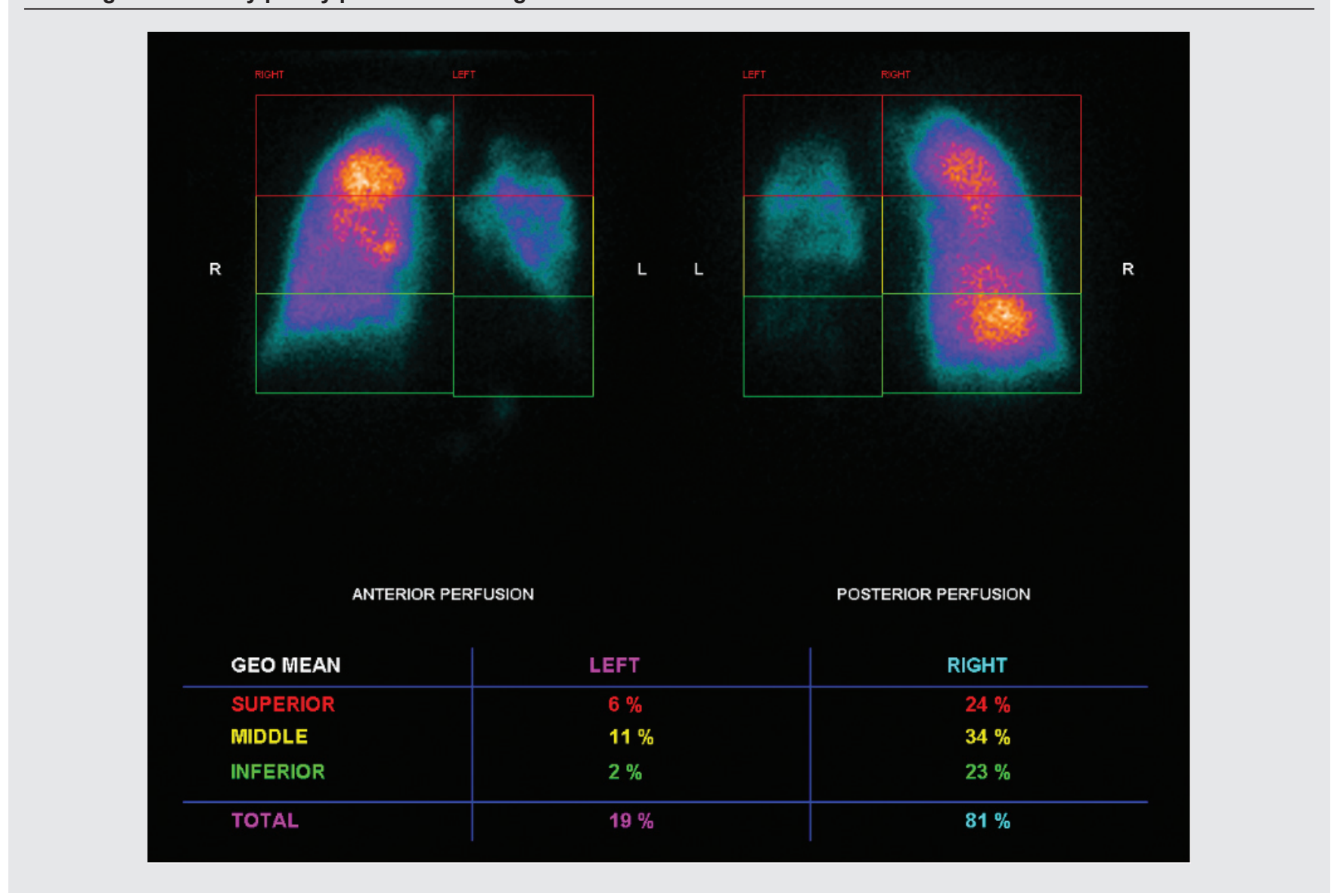

After completion of the bilateral lung lavage, the patient was reintubated with an endotracheal tube size 8.5 and the remaining fluid was removed from the lungs bronchoscopically. The sedated and mechanically ventilated patient remained hemodynamically stable and was admitted to the intensive care unit (ICU) with continued support of the VV-ECMO. Given the clinical stability and absence of complications, the patient was weaned off ECMO 2 hours after WLL. Lower-limb Doppler sonography was performed and deep venous thrombosis of the cannulated vessels excluded. The patient was extubated four hours after ICU admission and was discharged to the ward 24 hours later. He improved clinically and functionally with resolution of the respiratory failure ( $\mathrm{pO}, 71 \mathrm{mmHg}, \mathrm{FiO}, 21 \%$ ) and was discharged after two days with no further complications.

\section{DISCUSSION}

The evolution of PAP is variable and treatment modalities are limited. WLL remains the gold-standard therapy as it provides long-lasting benefits in most patients [12]. WLL is usually well tolerated, as shown in previous studies $[7,10,12]$, partially because auto-immune PAP is the most common form and patients normally do not have significant structural damage of lung parenchyma. In our center, the reference site for all patients that present with PAP in northern Portugal, the preferential treatment for PAP is sequential WLL with SLV. A total of 18 WLL have been performed with no major complications [7]. In this clinical case, however, PAP was associated with silicosis and bilateral consolidations, more extensive in the left lung, which was demonstrated by severe ventilation/perfusion impairment in lung scintigraphy. The patient wasn't able to tolerate SLV, because there is a greater risk of severe hypoxemia with this method. In these extremely rare cases of high-risk patients, ECMO-assisted WLL becomes a valid alternative [10, 13], as it allows the WLL to be performed without lung ventilation. We chose the VV-ECMO modality because of its lower rate of complications when compared with venoarterial ECMO [14]. Moreover, VV-ECMO could improve pulmonary arterial oxygenation, reducing pulmonary hypertension [15].

We agree with other reports about the importance of lavage being performed in both lungs in the same session as it improves the outcome [10], and we also believe it is safer to perform lavage of one lung at a time, as it minimizes the risk of hemodynamic instability. The saline infusion used for lavage was warmed up to $37^{\circ} \mathrm{C}$, to mitigate the risk of cardiac arrythmias, as shown in previous reports [16].

Despite its higher costs and associated risks, bilateral WLL with the support of VV-ECMO proved to be an effective and safe treatment in patients with PAP with severe hypoxemia that precludes the WLL method with SLV. Since these cases are extremely rare, published data are scarce and the ideal protocol has not yet been established. Future prospective studies are warranted.

\section{REFERENCES}

1. Rosen SH, Castleman B, Liebow AA. Pulmonary alveolar proteinosis. N Engl J Med 1958;258(23):1123-42. doi: 10.1056/NEJM 195806052582301

2. Suzuki T, Trapnell BC. Pulmonary alveolar proteinosis syndrome. Clin Chest Med 2016;37(3):431-40. doi: 10.1016/j.ccm.2016.04.006. 
3. McCarthy C, Avetisyan R, Carey BC, Chalk C, Trapnell BC. Prevalence and healthcare burden of pulmonary alveolar proteinosis. Orphanet J Rare Dis 2018;13(1):129. doi: 10.1186/s13023-018-0846-y.

4. Fijolek J, Wiatr E, Radzikowska E, et al. Pulmonary alveolar proteinosis during a 30-year observation. Diagnosis and treatment. Pneumonol Alergol Pol 2014;82(3):206-17. doi: 10.5603/PiAP.2014.0028.

5. Kumar A, Abdelmalak B, Inoue Y, Culver DA. Pulmonary alveolar proteinosis in adults: Pathophysiology and clinical approach. Lancet Respir Med 2018;6:554-65. doi: 10.1016/S2213-2600(18)30043-2.

6. Seymour JF, Presneill JJ. Pulmonary alveolar proteinosis: Progress in the first 44 years. Am J Respir Crit Care Med 2002;166(2):215-35. doi: $10.1164 / \mathrm{rccm} .2109105$.

7. Silva A, Moreto A, Pinho C, Magalhaes A, Morais A, Fiuza C. Bilateral whole lung lavage in pulmonary alveolar proteinosis - A retrospective study. Rev Port Pneumol 2014;20(5):254-9. doi: 10.1016/j. rppneu.2014.04.004.

8. Abdelmalak BB, Khanna AK, Culver DA, Popovich MJ.. Therapeutic whole-lung lavage for pulmonary alveolar proteinosis: A procedural update. J Bronchology Interv Pulmonol 2015;22(3):251-8. doi: 10.1097/ LBR.0000000000000180.

9. Roncon-Albuquerque R, Jr, Ferreira-Coimbra J, Vilares-Morgado R, Figueiredo P, Paiva JA. $\mathrm{PaO}_{2} / \mathrm{FiO}_{2}$ deterioration during stable extracorporeal membrane oxygenation associates with protracted recovery and increased mortality in severe acute respiratory distress syndrome. Ann Thorac Surg 2016;102(6):1878-85. doi: 10.1016/j.athoracsur.2016.06.026.
10. Sihoe AD, Ng VM, Liu RW, Cheng LC. Pulmonary alveolar proteinosis in extremis: The case for aggressive whole lung lavage with extracorporeal membrane oxygenation support. Heart Lung Circ 2008;17(1):69-72. doi: 10.1016/j.hlc.2006.11.007.

11. Roncon-Albuquerque R, Jr, Basilio C, Figueiredo P, et al. Portable miniaturized extracorporeal membrane oxygenation systems for H1N1-related severe acute respiratory distress syndrome: A case series. J Crit Care 2012;27(5):454-63. doi: 10.1016/j.jcrc.2012.01.008.

12. Beccaria M, Luisetti M, Rodi G, et al. Long-term durable benefit after whole lung lavage in pulmonary alveolar proteinosis. Eur Respir J 2004;23(4):526-31. doi: 10.1183/09031936.04.00102704.

13. Awab A, Khan MS, Youness HA. Whole lung lavage-technical details, challenges and management of complications. J Thorac Dis 2017;9(6): 1697-706. doi: 10.21037/jtd.2017.04.10.

14. Makdisi G, Wang IW. Extra Corporeal Membrane Oxygenation (ECMO) review of a lifesaving technology. J Thorac Dis 2015;7(7): E166-76.

15. Reis Miranda D, van Thiel R, Brodie D, Bakker J. Right ventricular unloading after initiation of venovenous extracorporeal membrane oxygenation. Am J Respir Crit Care Med 2015;191(3):346-8. doi: 10.1164/ rccm.201408-1404LE.

16. Sivitanidis E, Tosson R, Wiebalck A, Laczkovics A. Combination of extracorporeal membrane oxygenation (ECMO) and pulmonary lavage in a patient with pulmonary alveolar proteinosis. Eur J Cardiothorac Surg 1999;15(3):370-2. doi: 10.1016/S1010-7940(99)00013-5. 